\title{
The Vibration of Simply Supported Non-Uniform Cross Sectional Pipe Conveying Fluid Resting on Viscoelastic Foundation
}

\author{
MOHAMED GAITH \\ Faculty of Engineering Technology \\ Al-Balqa' Applied University \\ Marka, Amman \\ JORDAN
}

\begin{abstract}
The induced flexural vibration of slender pipe systems with continuous non uniform cross sectional area containing laminar flowing fluid lying on extended Winkler viscoelastic foundation is considered. The Euler Bernoulli model of the pipe has hinged ends. The inlet flow is considered constant steady that interacts with the wall of the pipe. The mathematical model is developed and its corresponding solution is obtained. The influence of the combination of variation of cross section, foundation stiffness and damping on the critical velocities, complex natural frequencies and stabilization of the system is presented.
\end{abstract}

Keywords: Conveying Pipe, Viscoelastic Foundation, Critical Velocity, Stability, Nonuniform Cross Section.

Received: May 4, 2020. Revised: August 26, 2020. Re-revised: September 10, 2020. Accepted: September 18, 2020. Published: September 21, 2020.

\section{Introduction}

Many researchers studied different parameters of the vibration of a pipe containing a flow fluid and presented the influence of these parameters in terms on the stability and critical flow velocity [1 -12]. In general, when the internal flow velocity is raised up to a definite critical value, the piping structure will be unstable. On the other hand, several studies investigated the effect of stiffness and damping of elastic foundation toward the stability features of the pipe containing flow fluid by obtaining the natural frequencies and critical flow velocities [13-25]. One of the early studies conducted by Stein et al. [18] who investigated the vibration features of a pipe containing pressurized flow fluid lying on an elastic foundation. They presented the effect of internal foundation stiffness, pressure, fluid velocity on the stability and can be guaranteed for certain range of flow velocities. Becker et al.[18] presented theoretical analysis for the vibration of pipes with fixed-free ends on different types of elastic foundation and validated with experimental results. Lottati et al.[23] investigated the influence of the foundation stiffness and damping on the vibration characteristics of the pipe conveying fluid for cantilever and fixed-fixed ends. They concluded that the effect of elastic foundation is revealed elevating of the critical flow fluid velocity. They found that damping is working as stabilizing or destabilizing dependent on the mass ratio of the pipe and fluid. Simply supported ends for a pipe containing flow fluid supported with elastic foundation was considered by Dermendjian-Ivanova [26]. He obtained the 
critical flow velocity and its dependence on the elastic foundation. Impollonia et al.[28] considered the articulated pipe containing flow fluid and demonstrated the influence of elastic foundations on divergence and flutter. Lilkova-Markova et al.[29] addressed the vibration characteristics of a pipe with fixed-free ends lying on an elastic foundation with lateral force at its tip. Carbon nanotubes containing flow fluid lying on viscoelastic Winkler elastic foundation was investigated by Ghavanloo et al.[30]. They presented the variation of resonant frequencies and critical flow velocities due to the presence of elastic and damping parameters in the Winkler foundation. Elishakoff et al.[31] and Djondjorov [25] considered elastic foundation partially attached to the cantilever pipe containing flow fluid and yet still can affect the stabilization of the piping system. Marzani et al.[32] considered a nonuniform elastic foundation of a cantilever pipe containing flow fluid. They concluded that the stability in terms of critical flow velocity can be affected depending on the mass ratio. Javadi et al. [33] considered the pipes of viscoelastic materials conveying fluid with viscoelastic of Kelvin-Voigt fractional type. Mostafa [34] used finite element method to analyze a pipe containing flow fluid with constant velocity lying on modified Winkler viscoelastic foundation and hinged at ends. He stated that the stiffness modulus tend to increase the critical flow velocities while damping acting in an opposite manner.

Olayiwola [21] utilized integral transforms to study the current piping system and presented closed form relations for vibration characteristics. Doare et al.[35] addressed the correlation of the local with global lateral motions of pipes containing flow fluid on Winkler elastic foundation and discussed its local and global instability. Karlicic et al.[36] conducted the nonlinear dynamic and vibration characteristics for single walled carbon nanotube in a magnetic field with simply supports at ends and lying on viscoelastic foundation of Kelvin-Voigt type. Skarian et al.[37] focused on a viscoelastic foundation modeled as fractional Kelvin-Voigt kind in investigating dynamic stability behavior of a pipe containing flow fluid. They showed that the mass ratio and fractional order parameter can be stabilizing or and destabilizing factors. Several studies considered Eddy current dampers to be used to suppress the dynamical instability [38-39 by Saxena et al.[14], Jae-Sung et al.[15] ]. Apparently, most of the above studies concluded that the stabilization effect of the stiffness foundation toward the features of pipe with internal flow such as the critical flow velocities and the critical resonant frequencies are proportionally increasing. The pipe conveying a fluid with variable cross sectional area has many applications in aircraft, biological engineering and industrial machines. Gaith [6] studied the vibration of pipe with variable cross section that is conveying a fluid hinged at ends and presented the impact of several parameters toward the stability map of the system. To the best of author's knowledge, the problem of pipe containing a flow fluid with nonuniform cross section and lying on an viscoelastic foundation was never considered before.

In this study lateral vibration in y-direction of pipe containing internal flow fluid with variable cross sectional and hinged at end is investigated. The governing equation of motion will be introduced analytically using energy and solved using Galerkin's procedure. The stability analysis of the piping system will be studied considering different input parameters.

\section{Formulation of The Problem}

Fig. 1 shows the pipe of finite length of $\mathrm{L}$ along the axial coordinate $\mathrm{x}$-direction, with inlet diameter $\boldsymbol{b}$, outlet diameter $\boldsymbol{a}$, mass per unit length $m_{p}(x)$ containing a flow fluid with constant inlet velocity $v_{o}$ and of mass per unit length $m_{f}(x)$. The pipe is of 
variable circular cross section and simply supported at ends, $\boldsymbol{E}$ is Young's constant, $I(x)$ is variable moment of inertia. The pip is lying on Winkler viscoelastic foundation of stiffness $\boldsymbol{k}$ and viscosity $\boldsymbol{c}$.

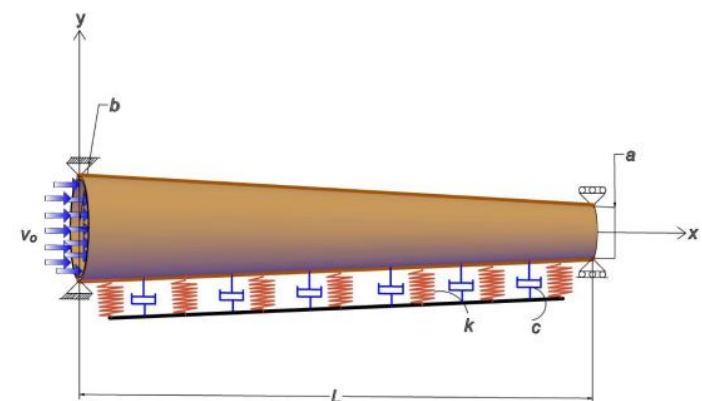

Figure 1. Schematic diagram of simply supported pipe with non-uniform cross section.

The partial differential governing equation of motion for the current piping system for the hinged ends is derived based on applying the variation procedure [5] of the total energy including kinetic and potential energies of the system and applying Hamilton's principle as following:

$E I \frac{\partial^{4} w}{\partial x^{4}}+k w+\left(2 E \frac{d I}{d x}\right) \frac{\partial^{3} w}{\partial x^{3}}+\left(E \frac{d^{2} I}{d x^{2}}+M V^{2}\right) \frac{\partial^{2} w}{\partial x^{2}}+$

$c \frac{\partial w}{\partial t}+2 M V \frac{\partial^{2} w}{\partial x \partial t}+(M+m) \frac{\partial^{2} w}{\partial t^{2}}+M V \frac{d V}{d x} \frac{\partial w}{\partial x}=0$

Where $w(x, t)$ is the flexural deflection of the pipe, $I(x)$ is the moment of inertia, $V(x)$ is the fluid velocity function of the axial span of the tubular beam, and the hinged ends conditions are defined as:

$$
\begin{aligned}
& I(x)=\frac{\pi}{4}\left\{\left(a+(b-a) \frac{x}{L}+h\right)^{4}-\left(a+(b-a) \frac{x}{L}\right)^{4}\right\} \\
& V(x)=v_{o} \frac{b^{2}}{\left(a+(b-a) \frac{x}{L}\right)^{2}} \\
& \left.w\right|_{x=0}=\left.\frac{\partial^{2} w}{\partial^{2} x}\right|_{x=0}=0, \text { and }\left.w\right|_{x=L}=\left.\frac{\partial^{2} w}{\partial^{2} x}\right|_{x=L}=0
\end{aligned}
$$

The parameters of the system can be presented in a non-dimensional form as following:

$$
\begin{aligned}
& W=\frac{w}{L}, \quad X=\frac{x}{L} \quad, \quad T=\frac{t}{L^{2}}\left(\frac{E I}{m_{f}+m_{p}}\right)^{\frac{1}{2}}, \\
& \bar{b}=\frac{b}{a}, \quad \bar{h}=\frac{h}{a}, \omega=\left(\frac{m_{f}+m_{p}}{E I}\right)^{\frac{1}{2}} L^{2} \Omega, \quad \mu=\frac{c L^{4}}{E I T}, \\
& K=\frac{k L^{4}}{E I}
\end{aligned}
$$

Hence, the governing equation of Eq.(1) is represented in non-dimensional form as:

$$
\begin{aligned}
& \frac{\partial^{4} W}{\partial X^{4}}+\frac{2}{\bar{I}} \frac{d \bar{I}}{d X} \frac{\partial^{2} W}{\partial X^{3}}+\left(\frac{1}{\bar{I}} \frac{d^{2} \bar{I}}{d X^{2}}+\frac{1}{\bar{I}} U^{2} A_{3}\right) \frac{\partial^{2} W}{\partial X^{2}}+ \\
& \frac{\partial^{2} W}{\partial T^{2}}+2 \beta^{\frac{1}{2}} U A_{1}\left(\frac{1}{\bar{I}}\right)^{\frac{1}{2}} \frac{\partial^{2} W}{\partial X}+U^{2} A_{2}\left(\frac{1}{\bar{I}}\right) \frac{\partial W}{\partial X}=0
\end{aligned}
$$

All the non-dimensional coefficients are listed in Appendix A. The general solution of the pipe deflection can be expressed in the form $W(X, T)=\varphi(X) e^{i \omega T}$. Hence, Eq. (6) becomes:

$\frac{d^{4} \varphi}{d X^{4}}+B_{1} \frac{d^{3} \varphi}{d X^{3}}+B_{2} \frac{d^{2} \varphi}{d X^{2}}+B_{3} \frac{d \varphi}{d X}+B_{4} \varphi=0$

The coefficients in Eq. (7) are listed in Appendix $\boldsymbol{A}$. The mode shape solution of the system can be calculated through the Galerkin's procedure assuming the mode function as:

$$
\varphi(X)=c_{1} \sin \pi X+c_{2} \sin 2 \pi X
$$

The complex circular frequency can be obtained using the four boundary conditions at hinged end.

\section{Results and Discussion:}

The pipe conveying a fluid with certain inlet velocity can stay in stable manner as long as it does not reach a certain value designated as critical flow velocity. Hence, Figs. 2 and 3 present the critical flow velocity for the first and second mode, respectively, for different non-uniform cross section ratios, $\boldsymbol{b} / \boldsymbol{a}$, at different pure foundation stiffness values. For a given specific stiffness value, 
the critical velocity is decreasing when increasing the cross section ratios. Meanwhile, the figures show clearly that by increasing the elastic foundation stiffness at a given cross section ratios, $\boldsymbol{b} / \boldsymbol{a}$, the corresponding critical velocity will increase. Since the cross section ratio is decreasing the critical velocity, adding elastic foundation will elevate the critical velocity significantly, and consequently the stable region will expand.

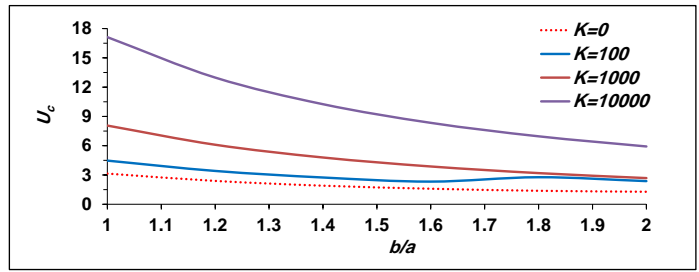

Figure 2. The dimensionless critical flow fluid velocity for the first resonant frequency versus the non-uniform cross section ratios, $\boldsymbol{b} / \boldsymbol{a}$ for different elastic foundation stiffness values $\boldsymbol{K}$ and $\mu=0$.

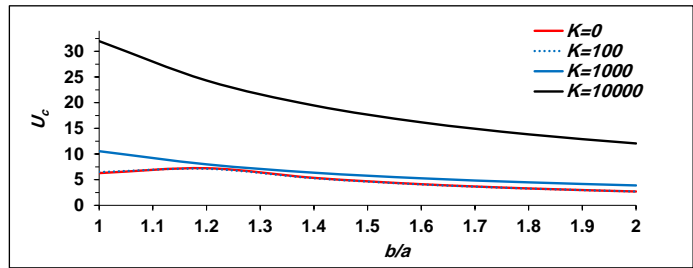

Figure 3. The dimensionless critical flow fluid velocity for the second resonant frequency versus the non-uniform cross section ratios, $\boldsymbol{b} / \boldsymbol{a}$ for different elastic foundation stiffness values $\boldsymbol{K}$ and $\mu=0$.

Figs. 4 and 5 show the influence of flow velocity on real parts of the first and second circular frequencies, respectively, at different values of foundation stiffness. The circular resonant frequencies are decreasing by increasing the flow velocity and diminish at certain critical flow velocity. For piping systems resting on an elastic foundation, the natural frequencies are elevated and vanish at higher critical flow velocity compared to the one without elastic foundation. This general conclusion is validated in previous studies for pipes with uniform cross sectional areas [18, 24,27].

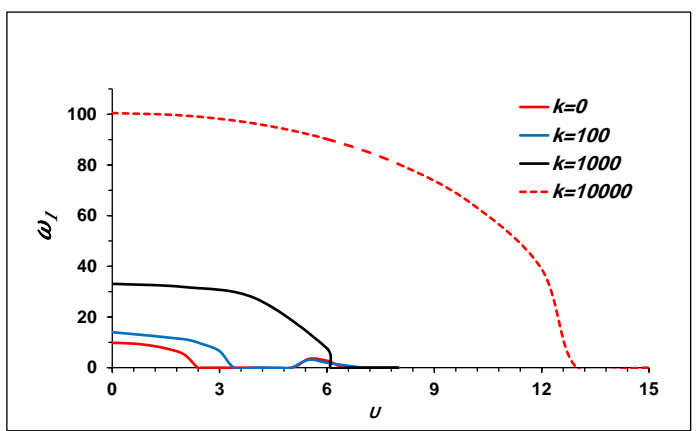

Figure 4. The dimensionless first circular resonant frequency versus the non-uniform flow fluid velocity, $\mathrm{U}$, for different elastic foundation stiffness values $\boldsymbol{K}, \mu=0$ and, $\boldsymbol{b} / \boldsymbol{a}$ $=1.2$.

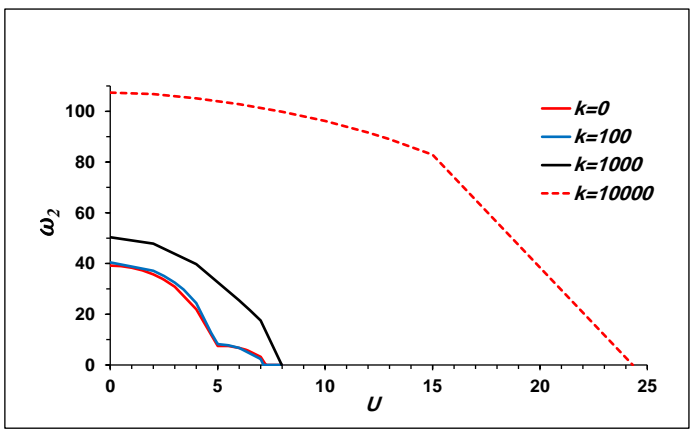

Figure 5. The non-dimensional second circular resonant frequency versus the nonuniform flow fluid velocity, $U$, for different elastic foundation stiffness values $\boldsymbol{K}, \mu=0$ and, $\boldsymbol{b} / \boldsymbol{a}=1.2$. Figure 5. The nondimensional second circular resonant frequency versus the non-uniform flow fluid velocity, $\mathrm{U}$, for different elastic foundation stiffness values $\boldsymbol{K}, \mu=0$ and, $\boldsymbol{b} / \boldsymbol{a}=1.2$.

The two complex parts of non-dimensional first and second natural frequencies are presented in Figs. 6 and 7, respectively, in terms of the fluid velocity for $K=0,100$ and damping $\boldsymbol{\mu}=0,30, \boldsymbol{b} / \boldsymbol{a}=1.2$. Fig. 6 shows that for $\boldsymbol{K}=0$ and 100 , with no foundation damping, $\boldsymbol{\mu}=\mathbf{0}$, there are real and imaginary parts for the second resonant frequency due to the cross section ratio, $\boldsymbol{b} / \boldsymbol{a}=1.2$. The real part is decreasing by increasing the flow velocity while the imaginary is almost zero. 
For $\boldsymbol{U}>4$, the real part is decreasing and the imaginary part is positively increasing which displays a contribution of positive damping effect. For $\boldsymbol{K}=100, \boldsymbol{\mu}=0$ and 30 . The existence of damping has lowered slightly the real parts and critical flow velocity but, concurrently, increased significantly the positive damping imaginary part for all flow velocities. Fig. 7 shows that the influence of foundation stiffness occurs in elevating the real part of first frequency and elevating the flow velocity till the real part is vanished. On the other hand, foundation damping is slightly affecting the stability region. Fig. 8 shows the real and imaginary parts of the second frequency for the case $\boldsymbol{K}=0,1000, \boldsymbol{\mu}=0,30$. The large value of foundation stiffness leads to huge elevation in the real part and elevates consequently the flow velocity where the real part vanishes. At that high stiffness value, the damping effect, $\boldsymbol{\mu}=30$, will elevate the imaginary damping part of the system for a certain flow velocity then it will decrease to zero at the critical velocity for it vanishes at $\boldsymbol{K}=1000$.

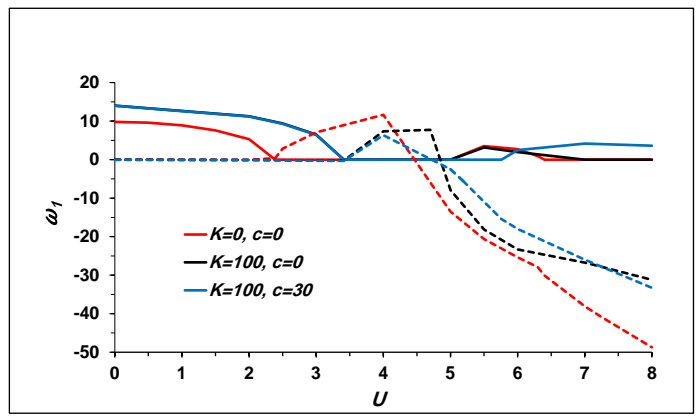

Figure 6. The real (solid) and imaginary (dashed) parts of dimensionless first resonant frequencies versus the dimensionless fluid velocity for different values of foundation stiffness $\boldsymbol{K}=0,100$ and damping $\boldsymbol{\mu}=0,30, \boldsymbol{b} / \boldsymbol{a}=1.2$.

Apparently the influence of damping on the first frequency is not substantial on the real and imaginary parts of the first circular frequency as shown in Fig. 9. However different behavior is displayed for the second circular frequency. Fig. 10 depicts the effect of foundation damping on the real and imaginary parts of the second circular frequency for $\mathrm{K}=100$ and $\mathrm{U}=2$. By increasing the foundation damping, the real part of second frequency is decreasing until it vanishes at $\mu=80$, and, concurrently, the imaginary part is increasing and having two positive damping branches at $\boldsymbol{\mu}=75$. Figures 11-13 display the influence of foundation damping on the two complex parts of second circular frequency for large stiffness values. Fig. 11 depicts the effect of damping on the real part of second circular frequency for large foundation stiffness $\boldsymbol{K}=$ $0,100,1000$, and 10000. The stiffness is elevating the real part and, and concurrently, the foundation damping $\boldsymbol{\mu}$ is

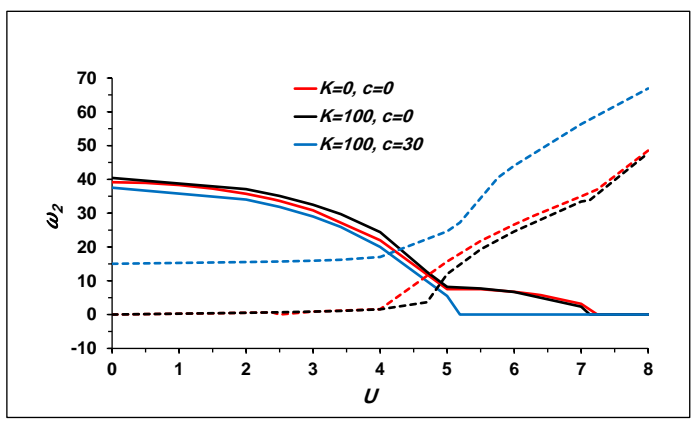

Figure 7. The real (solid) and imaginary (dashed) parts of dimensionless second resonant frequencies versus the dimensionless fluid velocity for variant values of foundation stiffness $\boldsymbol{K}=0,100$ and damping $\boldsymbol{\mu}=0,30, \boldsymbol{b} / \boldsymbol{a}=1.2$.

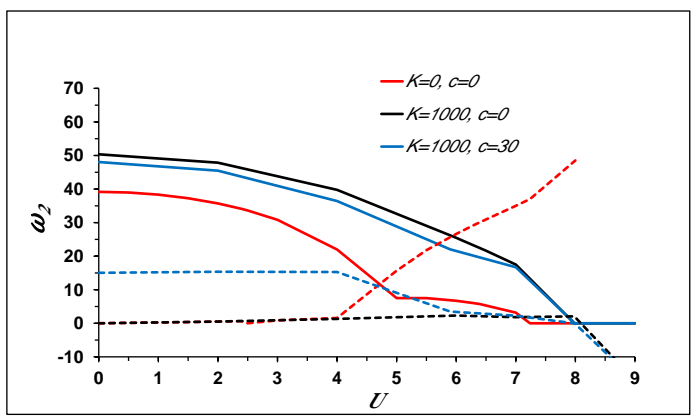

Figure 8. The real (solid) and imaginary (dashed) parts of dimensionless second resonant frequencies versus the dimensionless fluid velocity for variant values of foundation stiffness $\boldsymbol{K}=0,100$ and damping $\boldsymbol{\mu}=0,30, \boldsymbol{b} / \boldsymbol{a}=1.2$. 
reducing the real part. However, this foundation damping is playing a substantial role in building up the imaginary parts of the second frequency as shown in Figs. 12-13. The existence and increasing of foundation damping is lowering the real part and critical flow velocity, but is generating the imaginary damping part for the second frequency and, concurrently, the existence of foundation stiffness will generate two positive braches at larger foundation damping values. The first circular frequency is not affected significantly by the foundation damping.

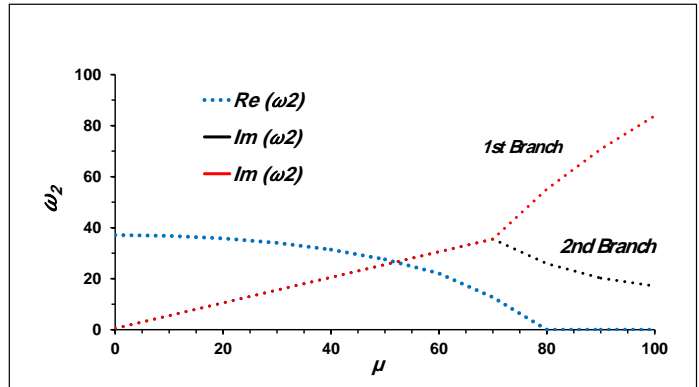

Figure 9. The relationship between the real and imaginary parts of dimensionless second resonant frequencies with the dimensionless foundation damping $\boldsymbol{\mu}$ for $\boldsymbol{K}=1000, \boldsymbol{U}=2$ and $\boldsymbol{b} / \boldsymbol{a}=1.2$.

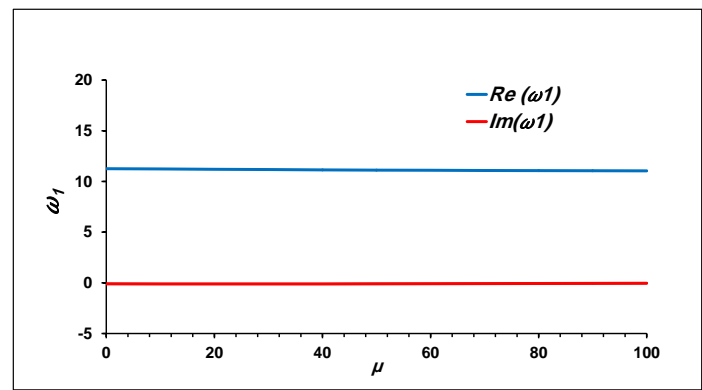

Figure 10. The relationship between the real (dashed) and imaginary (solid) parts of dimensionless second resonant frequencies with the dimensionless foundation damping $\boldsymbol{\mu}$ at $\boldsymbol{K}=100, \boldsymbol{U}=2$ and $\boldsymbol{b} / \boldsymbol{a}=1.2$.

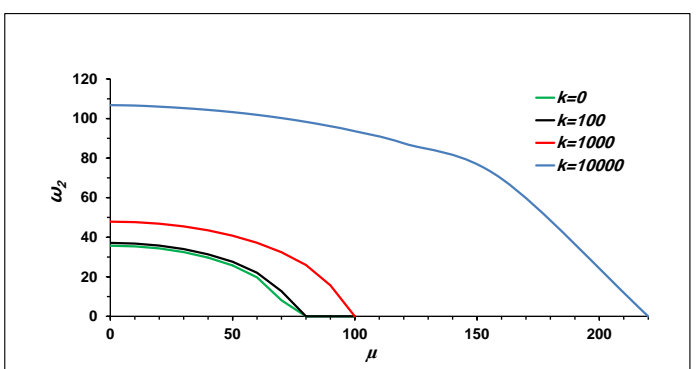

Figure 11. The relationship between the real part of dimensionless second resonant frequencies with the dimensionless foundation damping $\boldsymbol{\mu}$ for different large $\boldsymbol{K}$, at $\boldsymbol{U}=2$ and $\boldsymbol{b} / \boldsymbol{a}=1.2$.

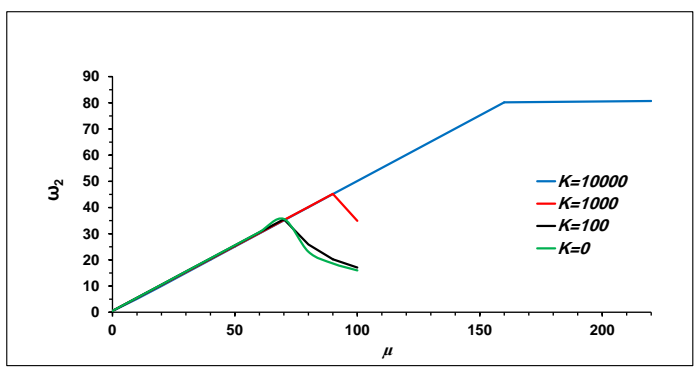

Figure 12. The relationship between the first imaginary part of dimensionless second resonant frequencies with the dimensionless foundation damping $\boldsymbol{\mu}$ for different large $\boldsymbol{K}$, at $\boldsymbol{U}=2$ and $\boldsymbol{b} / \boldsymbol{a}=1.2$.

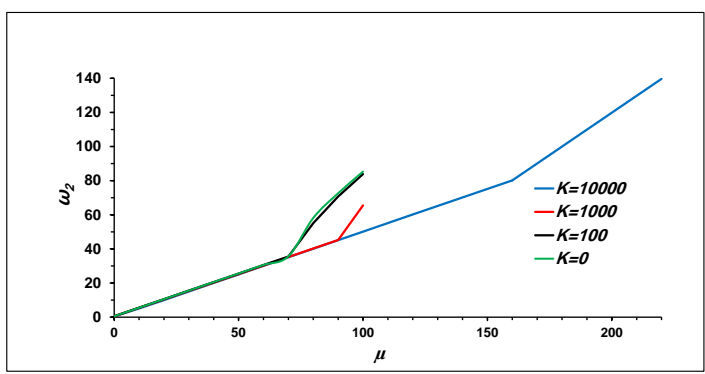

Figure 13. The relationship between the second imaginary part of dimensionless second resonant frequencies with the dimensionless foundation damping $\boldsymbol{\mu}$ for different large $\boldsymbol{K}$, at $\boldsymbol{U}=2$ and $\boldsymbol{b} / \boldsymbol{a}=1.2$. 


\section{Conclusion}

The effect of viscoelastic modified Winkler foundation and the non-uniform cross sectional area on the stability and vibration of a pipe with non-uniform cross section conveying a fluid with hinged ends is presented. It is found that the existence of non-uniform cross section will reduce the circular resonant frequencies and correlated critical flow fluid velocities, however, with the foundation stiffness; the natural frequencies are elevated and vanish at greater critical flow velocity compared to the one without elastic foundation. This will expand the stability regions and more significant for the second mode. The existence and increasing of foundation damping is lowering the real part and critical flow velocity, but is generating the imaginary damping part for the second frequency and, concurrently, the existence of foundation stiffness will generate two positive braches at larger foundation damping values. The first circular frequency is not affected significantly by the foundation damping.

\section{APPENDIX A}

$$
\begin{aligned}
& \beta=\frac{1}{1+\beta^{*}\left(\frac{2 \bar{h}}{(1+(\bar{b}-1) X}\right)} \\
& U=\left(\frac{\rho_{f} \pi}{E}\right)^{\frac{1}{2}} \frac{L v_{o}}{a^{2}} \\
& A_{1}=\frac{\bar{b}^{2}}{(1+(\bar{b}-1) X)^{1}} \\
& A_{2}=\frac{-2 \bar{b}^{4}(\bar{b}-1)}{(1+(\bar{b}-1) X)^{3}} \\
& A_{3}=\frac{\bar{b}^{4}}{(1+(\bar{b}-1) X)^{2}} \\
& \bar{I}=\frac{\pi}{4}\left\{(1+(\bar{b}-1) X+\bar{h})^{4}-(1+(\bar{b}-1) X)^{4}\right\}
\end{aligned}
$$

Where $\bar{h}$ is the dimensionless thickness of the pipe, $\Omega$ is the complex circular frequency and $\beta^{*}=\frac{\rho_{p}}{\rho_{f}}, \rho_{p}$ and,$\rho_{f}$ are density of pipe and fluid, respectively

$$
\begin{aligned}
& B_{1}=\frac{8(\bar{b}-1)}{(1+(\bar{b}-1) X)} \\
& B_{2}=\frac{12(\bar{b}-1)^{2}}{(1+(\bar{b}-1) X)^{2}}+U^{2} \frac{4 \bar{b}^{4}}{\pi(1+(\bar{b}-1) X)^{6}} \\
& B_{3}=2 \beta^{\frac{1}{2}} U\left(\frac{(2) \bar{b}^{2}}{\sqrt{\pi}(1+(\bar{b}-1) X)^{3}} i \omega+\right. \\
& U^{2} \frac{-8 \bar{b}^{4}(\bar{b}-1)}{\pi(1+(\bar{b}-1) X)^{7}} \\
& B_{4}=-\omega^{2} \frac{4}{\pi(1+(\bar{b}-1) X)^{4}}+k+\mu i \omega
\end{aligned}
$$

\section{References}

[1]. Païdoussis M., Fluid-structure interactions: slender structures and axial flow, vol. 1. London: Academic Press; 2014.

[2]. Li S., Karney B., Liu G., FSI Research in pipeline systems - a review of the literature, J Fluid Structure, Vol.57, 2015, pp. 277-97.

[3]. Li L., Hu Y., Critical flow velocity of fluid-conveying magneto-electro-elastic pipe resting on an elastic foundation, Int J Mech Sci, Vol.119, 2016, pp. 273-82.

[4]. Bahaadini R., Hosseini M., Nonlocal divergence and flutter instability analysis of embedded fluid-conveying carbon nanotube under magnetic field, Microfluid Nano fluidics, Vol.20(7), 2016, pp. 108.

[5]. Benjamin T., Dynamics of A system of Articulated Pipes Conveying Fluid .I. Theory, Proceedings of the Royal Society (London), A261, 1961, pp. 457486.

[6]. Gaith M., The dynamic response of tubular beam with variable cross section 
conveying fluid, International Journal of Mechanical Engineering \& Technology, Vol. 11, Issue 7, 2020, pp. 72-82.

[7]. Tang Y., Zhen Y., Fang B., Nonlinear vibration analysis of a fractional dynamic model for the viscoelastic pipe conveying fluid, Appl Math, Vol.56, 2018, pp. 123-36.

[8]. Firouz-Abadi R., Askarian A., Kheiri M., Bending-torsional flutter of a cantilevered pipe conveying fluid with an inclined terminal nozzle, Journal of Sound and Vibration, Vol.12, 2013, pp. 3002-14.

[9]. Yang X., Yang T., Jin J., Dynamic stability of a beam-model viscoelastic pipe for conveying pulsative fluid, Acta Mech Solida Sin, Vol.20(4), 2007, pp. $350-6$.

[10]. Hosseini M., Paparisabet M., The effects of blood flow on blood vessel buckling embedded in surrounding soft tissues, Int J Appl Mech, Vol.8, 2016, pp. 50065.

[11]. Zhao Y., Huang Y., Guo M., A novel approach for free vibration of axially functionally graded beams with non-uniform cross-section based on Chebyshev polynomials theory, Composite Structure, Vol.168, 2017, pp. 277-84.

[12]. Ibrahim R., Overview of mechanics of pipes conveying fluids, part I: fundamental studies, J. Press. Vessel Technol, Vol.32, 2010, pp. 1-32.

[13]. Djondjorov P., Vassilev V, Dzhupanov V, Dynamic stability of fluid conveying cantilevered pipes on elastic foundations, Journal of Sound and Vibration, Vol.247 (3), 2001, pp. 537-546.

[14]. Sinir B., Demir D., The analysis of nonlinear vibrations of a pipe conveying an ideal fluid, Eur J Mech B, Vol.52, 2015, pp. 38-44 .

[15]. Bahaadini R., Hosseini M, Nonlocal divergence and flutter instability analysis of embedded fluid conveying carbon nanotube under magnetic field,
Microfluid Nanofluidics, Vol.20(7), 2016, pp. 108.

[16]. Chellapilla K., Simha H., Critical velocity of fluid-conveying pipes resting on two-parameter foundation, Journal of Sound and Vibration, Vol.302, 2007, pp. 387-397.

[17]. Stein R., Tobriner M., Vibration of pipes containing flowing fluids, Trans ASME J Appl. Mechanics, 1970, pp. 906-916.

[18]. Becker M., Hauger W., Winzen W., Influence of internal and external damping on the stability of Beck's column on an elastic foundation, Journal of Sound and Vibration, Vol.54, 1977, pp. 468-472.

[19]. Kornecki L., The effect of an elastic foundation and of dissipative forces on the stability of fluid-conveying pipes, Journal of Sound and Vibration, Vol.109, 1986, pp. 327-338.

[20]. Liang F., Jin J., Yang X., Static and dynamic stabilities of fluid pipes on elastic foundation, Engineering Mechanics, Vol.11, 2010, pp. 166-171.

[21]. Olayiwola P., Mechanics of a FluidConveying Pipeline System Resting on a Viscoelastic Foundation, Journal of Multidisciplinary Engineering Science Studies, Vol. 2. Issue 3, 2016.

[22]. Fakher M., Hosseini-Hashemi S., Bending and free vibration analysis of nanobeams by differential and integral forms of nonlocal strain gradient with Rayleigh-Ritz method, Mater Res Express, Vol.4(12), 2017, pp. 125025.

[23]. Lottati I, Kornecki A, The effect of an elastic foundation and of dissipative forces on the stability of fluid conveying pipes, Journal of Sound and Vibration, Vol.109(2), 1986, pp. 327-338.

[24]. Li L, Hu Y, Critical flow velocity of fluid-conveying magneto-electro-elastic pipe resting on an elastic foundation, Int J Mech Sci, Vol.119, 2016, pp. 273-82.

[25]. Djondjorov P., Dynamic stability of pipes partly resting on Winkler foundation, Journal of Theoretical and Applied Mechanics, Vol. 31(3), 2001, pp. 101-112. 
[26]. Dermendjian-Ivanova D., Critical flow velocities of a simply supported pipeline on an elastic foundation, Journal of Sound and Vibration, Vol.157, 1992, pp. 370-374.

[27]. Ryu B., Ryu S., Kim G., Yim K., Vibration and dynamic stability of pipes conveying fluid on elastic foundations, KSME Int J, Vol.18(12), 2004, pp. 2148-57.

[28]. Impollonia N., Elishakoff I., Effect of Elastic Foundations on Divergence and Flutter of an Articulated Pipe Conveying Fluid, Journal of Fluids and Structures, Vol. 14, 2000, pp. 559-573.

[29]. Lilkova-Markova S., Lolov D., Cantilevered pipe conveying fluid, lying on Winkler elastic foundation and loaded by transversal force at the free end, J. Building (Sofia), Vol.4, 2003, pp. 5-8.

[30]. Ghavanloo E., Daneshmand F., Rafiei M., Vibration and instability analysis of carbon nanotubes conveying fluid and resting on a linear viscoelastic winkler foundation, Physica $E$, Vol.42(9), 2010, pp. 2218-24.

[31]. Elishakoff I., Impolonia N., Does a partial elastic foundation increase the flutter velocity of a pipe conveying fluid, Journal of Applied MechanicsTransactions of the ASME, Vol.68(2), 2001, pp. 206-212.

[32]. Marzani A., Mazzotti M., Viola E., Vittori P., Elishakoff I, FEM Formulation for Dynamic Instability of Fluid-Conveying Pipe on Nonuniform Elastic Foundation, Mechanics Based Design of Structures and Machines, Vol.40, 2012, pp. 83-95.

[33]. Javadi M., Noorian M., Irani S., Stability analysis of pipes conveying fluid with fractional viscoelastic model, Meccanica, Vol.54(3), 2019, pp. 399410.

[34]. Mostafa N., Effect of a Viscoelastic foundation on the Dynamic Stability of a Fluid Conveying Pipe, International Journal of Applied Science and Engineering, Vol.12, 2014, pp. 59-74.
[35]. Doare, E., De L., Local and global instability of fluid conveying pipes on elastic foundation, Journal of Fluids and Structures, Vol.16, 2002, pp. 1-14.

[36]. Karlicic D., Kozic P., Pavlovic R., Nesic N, Dynamic stability of singlewalled carbon nanotube embedded in a viscoelastic medium under the influence of the axially harmonic load, Composite Structure, Vol.162, 2017, pp. 227-43.

[37]. Askarian A., Permoon M., Shakouri M., Vibration analysis of pipes conveying fluid resting on a fractional Kelvin-Voigt viscoelastic foundation with general boundary conditions, International Journal of Mechanical Sciences, Vol.179, 2020, pp. 105702.

[38]. Saxena A., Patel C., Vibration Control of Cantilever Beam Using Eddy Current Damper, International Journal of Engineering and Innovative Technology, Vol. 2, Issue 3, 2013.

[39]. Jae-ung B., Moon K., Daniel I., Vibration Suppression of a Cantilever Beam Using Eddy Current Damper, Journal of Sound and Vibration, Vol.284, 2005, pp. 805-824.

\section{Creative Commons Attribution License 4.0 (Attribution 4.0 International, CC BY 4.0)}

This article is published under the terms of the Creative Commons Attribution License 4.0 https://creativecommons.org/licenses/by/4.0/deed.en_US 